\title{
Design and Realization of An Efficient Bio 9/7 Discrete Wavelet Transform
}

\author{
Zena N. Abdulkader \\ Computer Science Department, College of Education, University of Mosul, Mosul, Iraq. \\ zenatiq2@yahoo.com \\ Abstract
}

In this paper, the design of an efficient bio 9/7 filter bank for the discrete wavelet transform (DWT) is proposed. The design starts with the standard design constraints of the lifting scheme architecture. A modification in the resulting 9/7 filter bank coefficients after lifting is made leading to new sets of simple and less-number coefficients for both low-pass and high-pass branches of the direct structure of the DWT filter bank. In order to speed up the operations of the designed filter bank, the need for multipliers is eliminated by realizing the designed filter coefficients in a multiplier-less manner. That's each of the existing non-zero coefficient is represented as sum-of-powers-of-two (SOPOT). The complexity of resulting bio 9/7 filter bank structure is the lowest among other recent issued structures. The performance of resulting structure is then examined objectively by applying three different test images and computing the peak signal-to-noise-ratio (PSNR) values for the reconstructed image. Subjective evaluation of these images is also achieved by calculating the average mean opinion score (MOS). Objective and subjective evaluation indicate the eligibility of the designed bio 9/7 DWT filter bank for image processing.

Keywords: DWT, Bio 9/7 Filter Bank, Lifting Scheme, Polyphase, Perfect Reconstruction (PR), Sum-of-Powers-of-Two (SOPOT).

$$
\begin{aligned}
& \text { تصميم وتثفيذ تحويل مويجي منقطع كفوع نوع 9/7 ثنائي التعامد } \\
& \text { زينة ناطق عبد القادر } \\
& \text { قسم علوم الحاسوبـ كلية التربية. جامعة الموصلـ الموصلـ العراق }
\end{aligned}
$$

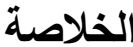

في هذا البحث، تم إقتراح تصميم كفوء لجرف المرشح العائد للتحويل المويجي المتقطع نوع 9/7 ثنائي التعامد.

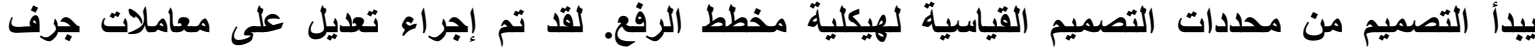

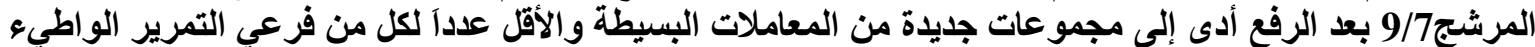

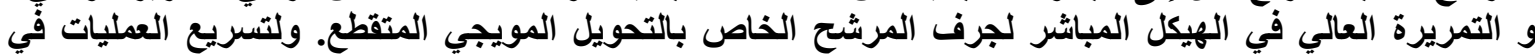

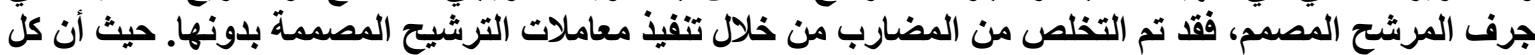

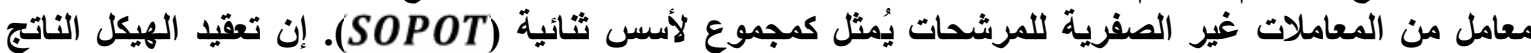

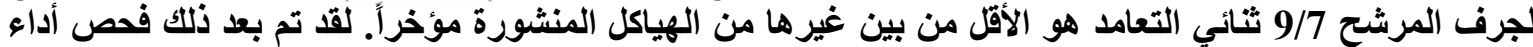

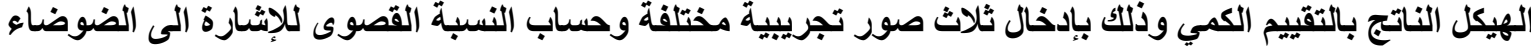

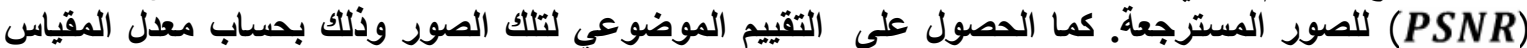
(MOS) المويجي المتقطع لمعالجة الصور. 


\section{Introduction}

The discrete wavelet transform (DWT) is developed as a multi-resolution transform that allows a good form of time-frequency analysis, which plays an important role in signal processing as it surmounts the limitation of solely time or frequency analysis [1], [2]. The DWT is widely used for signal and image analysis. Choosing a suitable wavelet filter structure is one of the major issues when using wavelet transforms [3], [4]. Many image and video compression systems include the DWT as the transformation stage. This paper deals with a simple design and efficient realization of symmetric biorthogonal CDF 9/7 wavelets.

The CDF 9/7 filter bank $(F B)$ of the biorthogonal 9/7 wavelet is proposed by Cohen, Daubechies, and Feauveau, [5]. The biorthogonal 9/7 wavelet have a great number of null moments for a relatively short support. They are more symmetrical and very close to orthogonality. This is an important feature in coding which ensures that the reconstruction error is very close to the quantization error in terms of mean squared error. The biorthogonal 9/7 wavelet transform is superior for the decorrelation of natural images and adopted as a core algorithm by the JPEG-2000 standard image coding being the default wavelet filters for lossy compression [6]. In spite of its wide range of applications, CDF 9/7 filters design method is too complicated and too complex [7]. In addition to that, the direct implementation method (i.e., convolution method) of such DWT usually requires a lot of arithmetic computations, since it is essentially a two-channel filter bank. Lifting scheme is another efficient way to reduce arithmetic complexity and to provide an in place implementation [8]. The Lifting scheme of the biorthogonal CDF 9/7 transform contains four steps: two prediction operators and two update operators [6].

Several designs was proposed for the efficient realization of the CDF 9/7 filter bank. In one of those optimal designs of biorthogonal wavelets that based on the lifting algorithm, Cheng, et al in 2003 [9]. A family of biorthogonal symmetric wavelet transforms was introduced by A. Z. Averbuch and V. A. Zheludev in 2004 utilizing splines for the design of filters for the predict and update operations in lifting schemes [10].They described the applications of such family to still image compression. Martina, et al in 2006 [5], constructed the compact support of biorthogonal wavelet filters and proposed a parametric expression for 9/7 wavelets. They approximated the bio 9/7 filter coefficients and expressed the $9 / 7$ filter outputs in terms of bio 5/3 filter outputs. By that approach, the adder-complexity of the 9/7 DWT was significantly reduced. In 2009, Quan and Ho proposed an efficient lifting scheme to construct biorthogonal wavelet [11] with better compression performance than the JPEG-2000 standard CDF 9/7 wavelet. Again in 2009, another design method was proposed for wavelet filter banks based on a single lifting structure suitable for $9 / 7$ filter pairs [12]. Those filters were derived directly without factorization. In such design, it was also shown that the signal boundaries can be treated without much computational efforts. A filter bank was included to be implemented in integer arithmetic without divisions. The designed wavelet filter banks showed better performance than the standard 9/7 filter bank for lossless image compression, while a competitive performance was achieved when they were applied in lossy compression.

In 2011 an efficient implementation of the DWT using polyphase filter bank architecture with distributed arithmetic $(D A)$ to speedup wavelet computation was proposed by G. Tewari, et al, [13]. That DWT was irreversible and utilized the 9/7 coefficients for a lossy compression. Also in 2011, M. Beladgham, et al [6] used the biorthogonal CDF 9/7 wavelet compression based on lifting scheme, coupled with the 
SPIHT coding to improve the quality of the compressed medical image. Recently, S. Zhang, et al, [7] suggested a 9/7 wavelet filter bank for texture image coding applications based on lifting a bio 5/3 filter to a bio $7 / 5$ filter, and then to a bio $9 / 7$ filter. A onedimensional optimization problem for such bio $9 / 7$ filter family was also carried out according to the perfect reconstruction $(P R)$ condition of wavelet transforms and wavelet properties. In addition, a suitable control parameter of the bio $9 / 7$ filter family for image coding applications was then determined. More recently, multiplier-less 1-D and 2-D DWT using bio 9/7 filter based on a new efficient distributed arithmetic (NEDA) technique was given by A. P. Gupta in [14].

This paper introduces a simple and efficient design leads to a multiplier-less realization of the filter bank of the symmetric biorthogonal CDF $9 / 7$ wavelet. A modification in the standard design constraints of the lifting scheme architecture leads to sets of simple and less-number filter coefficients. These coefficients possess some interesting characteristics which ease the overall filter bank realization. That is they can be easily realized in a multiplier-less manner using the method of sum-of-powers-of-two (SOPOT). The resulting multiplier-free structure is compared with some other recent ones. The symmetric biorthogonal CDF 9/7 wavelet transform of the designed type is also tested through analyzing and synthesizing some standard images highlighting its applicability.

The rest of this paper is organized as follows; The background of the bio 9/7 wavelet filter bank is given in section 2. The proposed design is presented in section 3 . In section 4 , efficient multiplier-less representations of the designed coefficients are explained. A comparative study concerning the basic building blocks of the bio $9 / 7$ filter bank is illustrated in section 5. The resulting structure is examined and analyzed in section 6 by computing the peak signal-to-noise-ratio (PSNR) values of some tested images. Finally, section 7 concludes this paper.

\section{The Background of the Bio 9/7 Wavelet Filter Bank}

In the filter bank of the biorthogonal 9/7 shown in Fig. 1 (analysis side), the transfer function of the low pass filter $H(Z)$ contains 9 coefficients and the transfer function of the high pass filter $G(Z)$ contains 7 coefficients. These anti-causal transfer functions are given by

$H_{(z)}=h_{-4} z^{4}+h_{-3} z^{3}+h_{-2} z^{2}+h_{-1} z+h_{0}+h_{1} z^{-1}+h_{2} z^{-2}+h_{3} z^{-3}+h_{4} z^{-4}$

and

$G(z)=g_{-3} z^{3}+g_{-2} z^{2}+g_{-1} z+g_{0}+g_{1} z^{-1}+g_{2} z^{-2}+g_{3} z^{-3}$

For symmetrical biorthogonal $9 / 7$ filters (i. e., $h_{i}=h_{-i}$, for $i=0,1,2,3,4$ and $g_{i}=g_{-i}$, for $\left.i=0,1,2,3\right), H_{(Z)}$ will be left only with 5 coefficients, while the high pass filter $G(z)$ will have 4 coefficients only, as follows:

$$
\begin{aligned}
& H(z)=h_{4}\left(z^{4}+z^{-4}\right)+h_{3}\left(z^{3}+z^{-3}\right)+h_{2}\left(z^{2}+z^{-2}\right)+h_{1}\left(z+z^{-1}\right)+h_{0} \\
& \text { and } \\
& G(z)=g_{3}\left(z^{3}+z^{-3}\right)+g_{2}\left(z^{2}+z^{-2}\right)+g_{1}\left(z+z^{-1}\right)+g_{0}
\end{aligned}
$$


The direct realizations of (3) and (4) can decrease the number of multiplications into approximately half its value using the advantage of the symmetric coefficients (i.e., linear phase property of each filter). For $P R$ condition, the filters of the synthesis side of Fig. 1 are chosen as

$\widetilde{H}(Z)=G(-Z)$

and

$\tilde{G}(z)=H_{(-Z)}$

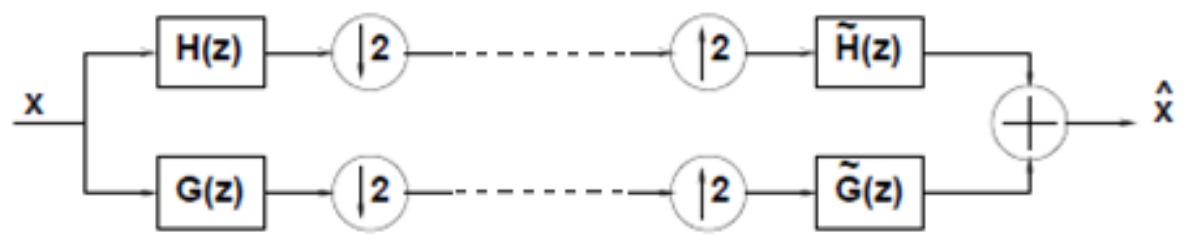

Fig. 1 Filter bank block scheme used to realize 1-D DWT.

In polyphase realization, each of the filters $H(z)$ and $G(Z)$ of the analysis bank is divided into even parts $H_{e}(Z) \& G_{e}(Z)$ and odd parts $Z^{-1} H_{o}(Z) \& Z^{-1} G_{o}(Z)$ as follows:

$$
\begin{aligned}
& H_{(Z)}=H_{e}(Z)+z^{-1} H_{o}(Z) \\
& \text { and } \\
& G_{(Z)}=G_{e}(Z)+z^{-1} G_{o}(Z) \\
& \text { where } \\
& H_{e}(Z)=h_{4}\left(z^{4}+z^{-4}\right)+h_{2}\left(z^{2}+z^{-2}\right)+h_{0} \\
& H_{o}(Z)=h_{3}\left(z^{4}+z^{-2}\right)+h_{1}\left(z^{2}+1\right) \\
& \left.G_{e} r z\right)=g_{2}\left(z^{2}+z^{-2}\right)+g_{0} \\
& \text { and } \\
& G_{o}(Z)=g_{3}\left(z^{4}+z^{-2}\right)+g_{1}\left(z^{2}+1\right)
\end{aligned}
$$

The samples of the input sequence $x(\mathrm{n})$ in Fig. 1 are initially de-multiplexed into even samples $x_{\text {even }}$ and odd samples $x_{\text {odd }}$. The even samples are convolved with the even filter coefficients, while the odd samples (after a single delay) are convolved with the odd filter coefficients. Finally, the even and odd phases are summed together [15]. Using the advantage of decimation in polyphase realization, the number of multiplication operations is further decreased by half. Thus, both decimation operation and linear phase property can reduce number of multiplication operations to $1 / 4$ of its total required. Lifting scheme is the latest efficient realization method to reduce the arithmetic complexity and to provide an in place implementation [8]. In the next section, the proposed design is derived using such scheme as a starting point.

\section{The Proposed Design}

As shown in Fig. 2, the lifting schemes consist of following 3-steps [16]: the splitter- where the input sequence is separated into even and odd parts, the predict stepwhere the even samples are multiplied by the time-domain equivalent of $T(z)$ and then added to odd samples and the update step- where the predict samples are multiplied by time-domain equivalent $S(z)$ and then added to even samples. 


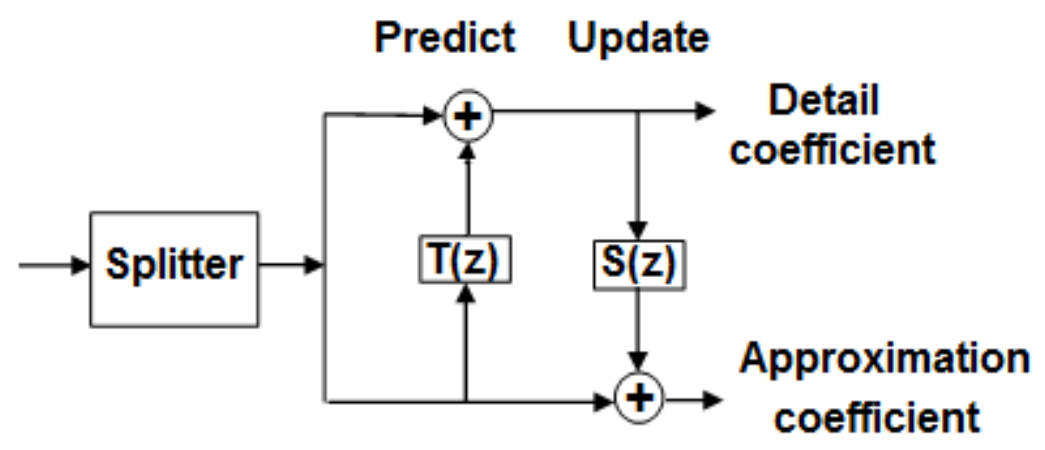

Fig. 2 The Lifting scheme steps.

The corresponding polyphase matrix factorization is given by [16]:

$$
\mathrm{P}(\mathrm{z})=\prod_{\mathrm{k}=1}^{\mathrm{n}}\left[\begin{array}{cc}
1 & \mathrm{~S}(\mathrm{z}) \\
0 & 1
\end{array}\right]\left[\begin{array}{cc}
1 & 0 \\
\mathrm{~T}(\mathrm{z}) & 1
\end{array}\right]
$$

The basic function of the lifting scheme method is to factorize the polyphase matrix of wavelet filter into a sequence of alternating upper and lower triangular matrices and diagonal matrices. According to [7], a new polyphase matrix can be formulated as follows:

or

$$
P^{\text {new }}(Z)=P(Z)\left[\begin{array}{cc}
1 & S(z) \\
0 & 1
\end{array} \mid\right.
$$

$$
P^{n e w}(Z)=\left[\begin{array}{ll}
H_{e}(z) & H_{e}(z) S(Z)+G_{e}(z) \\
H_{O}(z) & H_{O}(z) S(Z)+G_{O}(z)
\end{array} \mid\right.
$$

According to the perfect reconstruction condition of 9/7 wavelet transform, wavelet properties, and normalizing condition, the above coefficients can be expressed in the form of a one-dimensional function. Thus, the resulting 9/7 coefficients after lifting are given as [7]

$$
\begin{aligned}
& h_{0}=-\frac{\left(8 t^{3}-18 t^{2}+7 t-20\right)}{16 t} \\
& h_{1}=\frac{\left(4 t^{3}-11 t^{2}+15 t-4\right)}{8 t} \\
& h_{2}=\frac{(t-2)}{4 t} \\
& h_{3}=\frac{\left(4 t^{2}-7 t+4\right)(t-1)}{8 t} \\
& h_{4}=\frac{\left(4 t^{2}-7 t+4\right)(2 t-1)}{32 t} \\
& g_{0}=\frac{(t+1)}{4} \\
& g_{1}=-\frac{(2 t+7)}{32} \\
& g_{2}=-\frac{(t-1)}{8} \\
& g_{3}=\frac{(2 t-1)}{32}
\end{aligned}
$$


where the range of $t$ can be determined as $t \subset[0.78,1.85]$. If $t$ is a known number, the filter coefficients can be easily determined using (16). While normalizing the frequency response at $\omega=0 \mathrm{rad}$. (i.e., $\left.H\left(e^{j \omega}\right)\right|_{\omega=0}=1$ and letting $t=1$ in (16), the following coefficient values can be obtained:

$$
\begin{array}{ll}
h_{0}=\frac{23}{32} & \mid \\
h_{1}=\frac{1}{4} & \mid \\
h_{2}=-\frac{1}{8} & \mid \\
h_{3}=0 & \mid \\
h_{4}=\frac{1}{64} & \\
g_{0}=\frac{1}{2} & \mid \\
g_{1}=-\frac{9}{32} & \mid \\
g_{2}=0 & \mid \\
g_{3}=\frac{1}{32} & J
\end{array}
$$

By substituting the resulting coefficient values $h_{i}$ for $i=0,1,2,3,4$ and $g_{i}$ for $i=$ $0,1,2,3$ in (9) to (12), The resulting transfer functions $H(z)$ and $G(z)$ in (7) and (8), respectively can be achieved by evaluating the followings:

$$
\begin{aligned}
& H_{e}(Z)=\frac{1}{64}\left(Z^{4}+Z^{-4}\right)-\frac{1}{8}\left(Z^{2}+Z^{-2}\right)+\frac{23}{32} \\
& H_{o}(Z)=\frac{1}{4}\left(Z^{2}+1\right) \\
& G_{e}(Z)=\frac{1}{2}
\end{aligned}
$$

and

$G_{o}(Z)=\frac{1}{32}\left(Z^{4}+z^{-2}\right)-\frac{9}{32}\left(z^{2}+1\right)$

It is necessary to point out that the resulting $9 / 7$ filter bank is of the wavelet type., since the regularity of the designed $9 / 7$ wavelet is guaranteed by the same test of [9] while the coefficients can be derived using the vanishing moments that satisfy $M=2$ and $M=4$. It can be calculated using

$$
\left.\begin{array}{ll}
\left.H^{(k)}(z)\right|_{\mathrm{z}=-1}=0 & \text { for } k=0,1 . \\
\left.G^{(k)}(z)\right|_{\mathrm{z}=-1}=0 & \text { for } k=0,1,2,3 .
\end{array}\right\}
$$

It should be noted that for different vanishing moments conditions and different normalization conditions, different coefficients can be evaluated. The resulting coefficient values in (18) - (21) are investigated for efficient multiplier-less realization in the next section. Impulse responses $h(n)$ and $g(n)$ of the proposed bio 9/7 filter bank are shown in Figs. 3 and 4 with their frequency responses $H\left(e^{j \omega}\right)$ and $G\left(e^{j \omega}\right)$ being illustrated in Figs. 5 and 6 respectively. Figures 5 and 6 also contain plots of the frequency responses of the original bio 9/7 filters. From these figures, it can be seen that the frequency responses of the proposed filter bank can approximate the originals, especially at pass-band range which is obviously much important than the stop-band. 


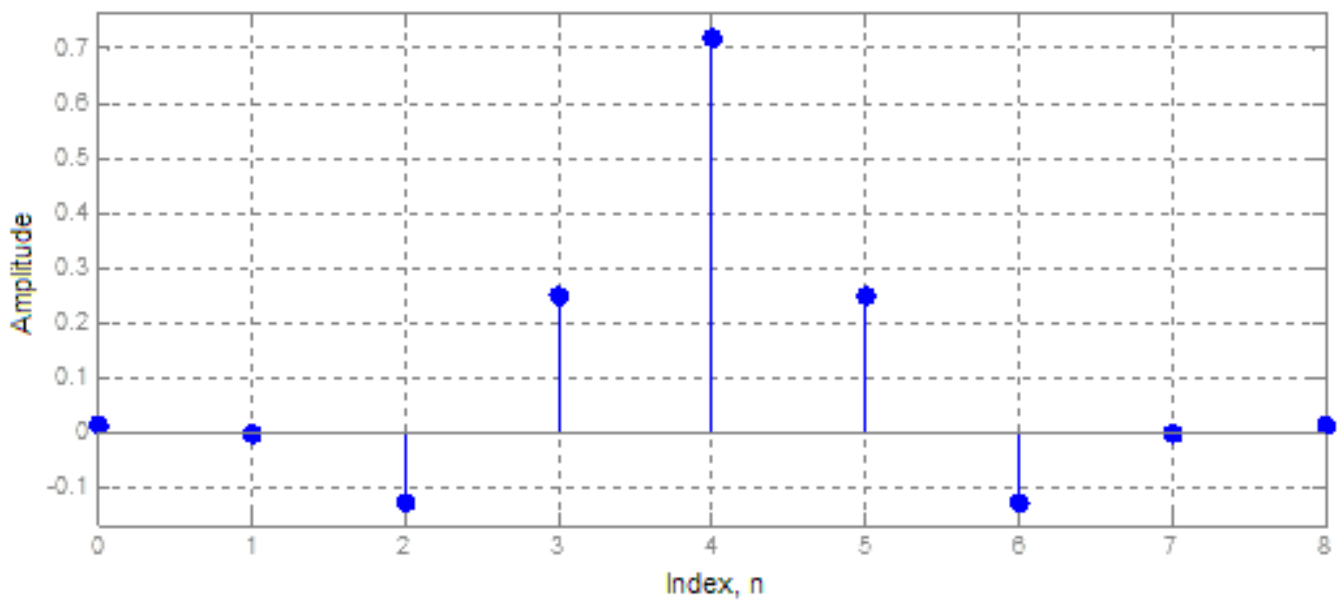

Fig. 3 Impulse response $h(n)$.

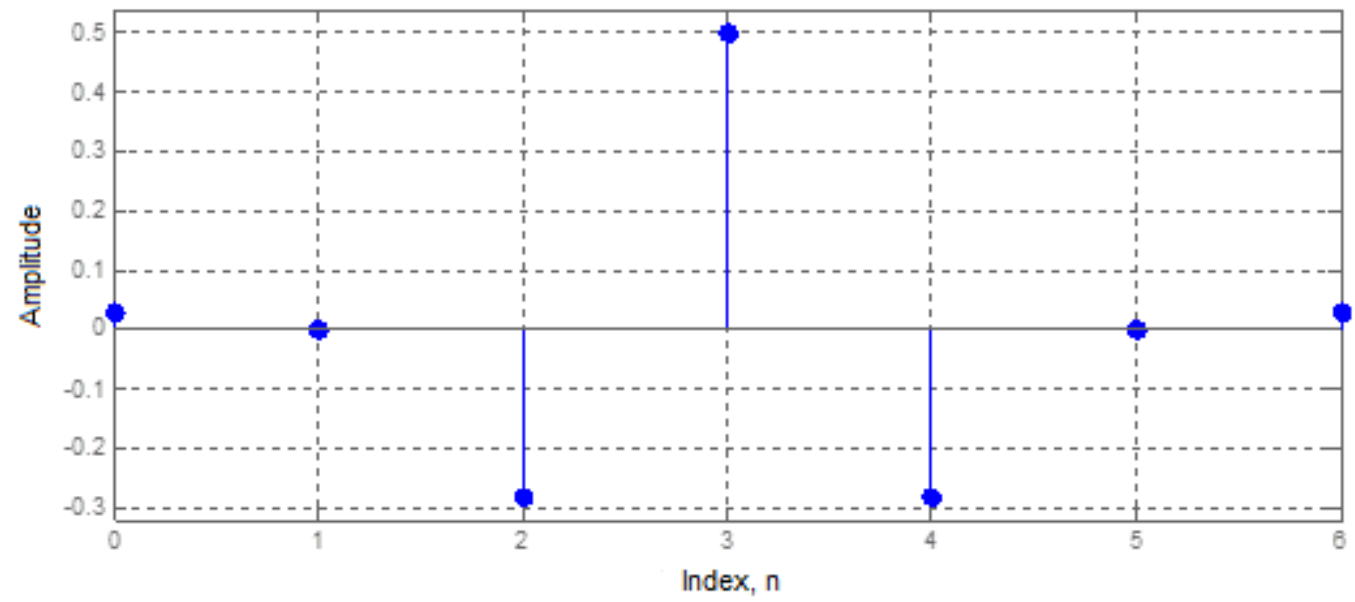

Fig. 4 Impulse response $g(n)$.

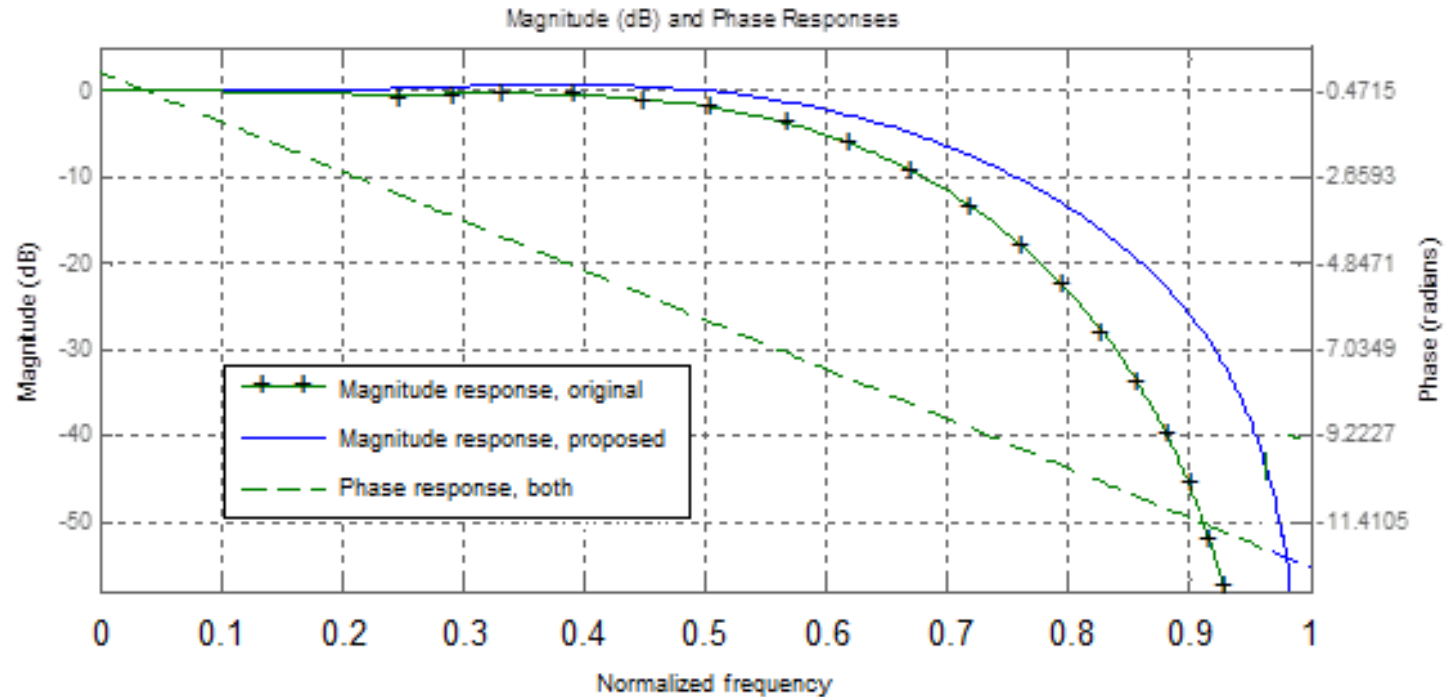

Fig. 5 Magnitude and phase responses of $H\left(e^{j \omega}\right)$, original and proposed. 


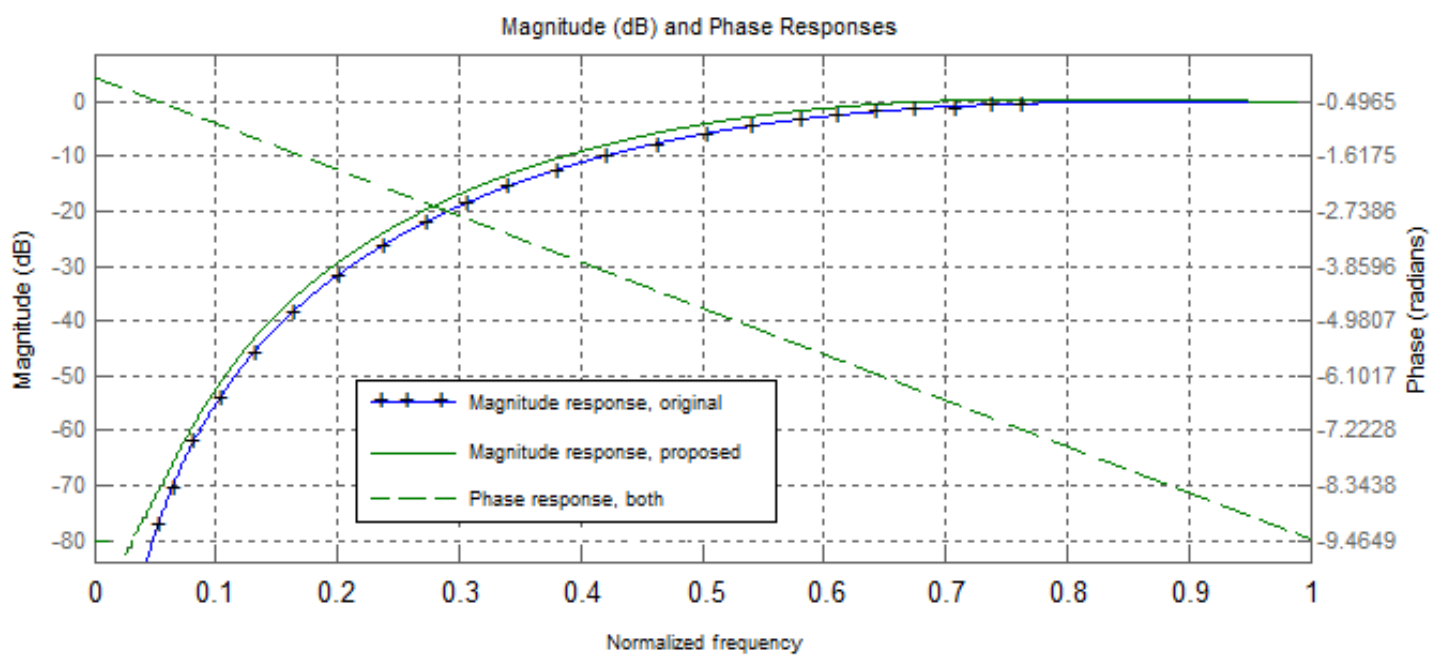

Fig. 6 Magnitude and phase responses of $G\left(e^{j \omega}\right)$, original and proposed.

The resulting bio 9/7 wavelet analysis one-dimensional (1-D) filter bank structure is shown in Fig. 7. Furthermore, in Fig. 7, the even function filtering sections $H_{e}(Z), H_{o}(Z)$, $G_{e}(Z)$ and $G_{o}(Z)$, followed by a down-sampler is equivalent to have a down-sampler first, then followed by half-ordered versions of those functions. Because of such lower sampling rate utilization, saving in computations by a factor of 2 will be achieved [17]. The resulting efficient filter bank structure is shown in Fig. 8. A two-dimensional (2-D) structure of Fig. 8 can easily constructed by repeating that figure three times in a tree structure.

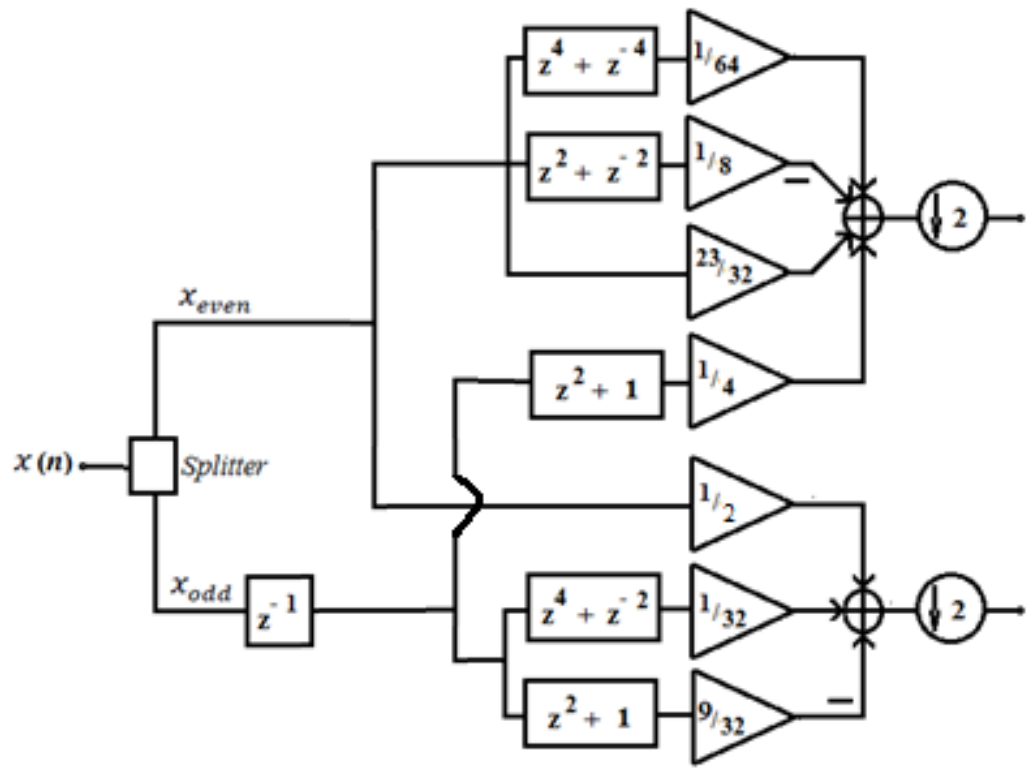

Fig. 7 The resulting bio 9/7 wavelet analysis one-dimensional (1-D) filter bank structure. 


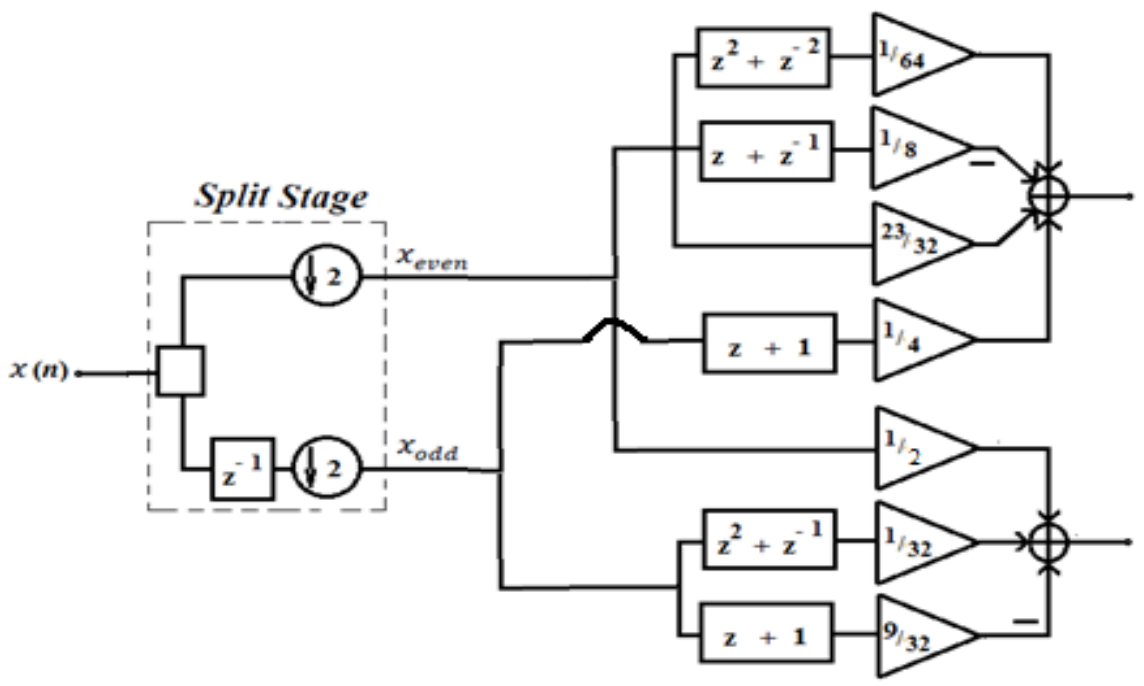

Fig. 8 Bio 9/7 wavelet analysis one-dimensional (1-D) filter bank structure with saving in computations by almost a factor of 2 .

\section{Efficient Multiplier-less Representation of the Designed Coefficients}

In addition to the pre-mentioned saving in computations in Fig. 8 by almost half of its original value, two reductions in realization complexity can also be achieved. In that context, it is known that multiplier representation takes a large cost in the utilized area and can reduce the speed of those systems which contain a large number of multipliers. This in turn reduces the maximum operating frequency. To eliminate the need for multipliers in the proposed architecture realization and in order to speed up its operation, the designed filter coefficients are realized in this paper in a multiplier-less manner. Two reductions in realization complexity of Fig. 8 can be achieved by a two-step manipulation in the bio 9/7 filter bank realization. The first step here is to make the design efficient by reducing (by 4) the number of coefficients required to represent each filter in the 9/7 filter bank. This was done by setting $t=1$ in (16) leading to $h_{3}=h_{-3}=g_{2}=g_{-2}=0$. The second step is accomplished by realizing each of the existing non-zero coefficient in (18)-(21) as sum-ofpowers-of-two (SOPOT) [18]. Multiplications are then achieved by only shift and add or even by a shift only. The values of the coefficients in the designed bio $9 / 7$ wavelet filter bank of Fig. 8 can be represented as a SOPOT as in Table 1.

Table 1 Multiplier-less representations of coefficients.

\begin{tabular}{||c||c||c|c||}
\hline $\begin{array}{c}\text { Coefficients of } \\
H(Z)\end{array}$ & $\begin{array}{c}\text { SOPOT } \\
\text { Representations }\end{array}$ & Coefficients of $G(Z)$ & $\begin{array}{c}\text { SOPOT } \\
\text { Representations }\end{array}$ \\
\hline$h_{0}=\frac{23}{32}$ & $\begin{array}{c}2^{0}-2^{-2}-2^{-5} \\
\text { Shift and add }\end{array}$ & $g_{0}=\frac{1}{2}$ & $\begin{array}{c}2^{-1} \\
\text { Shift only }\end{array}$ \\
\hline$h_{1}=h_{-1}=\frac{1}{4}$ & $\begin{array}{c}2^{-2} \\
\text { Shift only }\end{array}$ & $g_{1}=g_{-1}=-\frac{9}{32}$ & $\begin{array}{c}-2^{-5} \\
\text { Shift and add }\end{array}$ \\
\hline$h_{2}=h_{-2}=-\frac{1}{8}$ & $\begin{array}{c}-2^{-3} \\
\text { Shift only }\end{array}$ & $g_{2}=g_{-2}=0$ & Non \\
\hline$h_{3}=h_{-3}=0$ & Non & $g_{3}=g_{-3}=\frac{1}{32}$ & $\begin{array}{c}2^{-5} \\
\text { Shift only }\end{array}$ \\
\hline$h_{4}=h_{-4}=\frac{1}{64}$ & $\begin{array}{c}2^{-6} \\
\text { Shift only }\end{array}$ & \multicolumn{2}{|c}{} \\
\hline
\end{tabular}




\section{A Comparative Study}

1-D and 2-D structures belongs to Fig. 8 are compared with other recent multiplierfree structures, namely; 2-D one of G. Tewari, et al in 2011 [13] and 1-D and 2-D structures of A. P. Gupta, et al in 2013 [14]. Table 2 illustrates this comparison. The comparison includes the numbers of required adders and shift registers. As illustrated in Table 2, the proposed structure (in 1-D and 2-D forms) outperforms the others in terms of all basic building blocks.

Table 2 A comparison between the proposed and other recent multiplier-free structures.

\begin{tabular}{|c|c|c|}
\hline Structure & $\begin{array}{c}\text { No. of } \\
\text { Adders }\end{array}$ & $\begin{array}{c}\text { No. of } \\
\text { Shift Registers }\end{array}$ \\
\hline G. Tewari, et al (2-D) [13] in 2011 & 80 & 68 \\
\hline A. P. Gupta, et al (1-D) [14] in 2013 & 36 & 24 \\
\hline A. P. Gupta, et al (2-D) [14] in 2013 & 80 & 72 \\
\hline Proposed Structure (1-D) & 9 & 13 \\
\hline Proposed Structure(2-D) & 27 & 39 \\
\hline
\end{tabular}

\section{Experimental Results}

The performance of the proposed bio 9/7 wavelet filters is measured and compared with those of the original bio 9/7 wavelet filters by applying three standard test images as inputs. PSNR values of the resulting reconstructed images are then calculated. High PSNR vales indicate that the output images are very similar to their originals copies. For an 8-bit $R \times C$-sized gray scale output image, the PSNR can be computed (in $\mathrm{dBs)}$ as follows [15]:

$P S N R=10 \log _{10}\left(\frac{255^{2}}{M S E}\right)$

Where $M S E$ is the mean square estimated

$\left.M S E=\frac{1}{R \times C} \sum_{r=0}^{R-1 C-1}\right\rangle_{c=0}\left|I_{o}(r, c)-I_{i}(r, c)\right|^{2}$

$I_{i}(r, c)$ is the input image and $I_{o}(r, c)$ is the reconstructed image. Table 3 illustrates the $M S E$ and PSNR values of three reconstructed test images shown in Fig. 9 (namely; Lena, Peppers and House) obtained using original and proposed bio 9/7 wavelet filters. The high $P S N R$ values highlight the $P R$ property of the designed wavelet filter bank. From Table 3, it can be easily noticed that the proposed bio $9 / 7$ wavelet filters can achieve all the three reconstructed test images with better quality than those of the originals presented in [14].

Table $3 M S E$ and $P S N R$ values of three reconstructed images using original and proposed bio 9/7 wavelet filters.

\begin{tabular}{|c|c|c|c|c|}
\hline \multirow[t]{2}{*}{ Image } & \multicolumn{2}{|c|}{$\begin{array}{c}\text { Results using Original } \\
\text { bio } 9 / 7 \text { wavelet filters [14] }\end{array}$} & \multicolumn{2}{|c|}{$\begin{array}{l}\text { Results using the proposed } \\
\text { bio } 9 / 7 \text { wavelet filters }\end{array}$} \\
\hline & $M S E$ & $P S N R(\mathrm{~dB})$ & $M S E$ & $P S N R(\mathrm{~dB})$ \\
\hline Lena $(512 \times 512)$ & 1.7047 & 45.8142 & 1.5273 & 46.2917 \\
\hline Peppers $(512 \times 512)$ & 2.0757 & 44.9591 & 1.8877 & 45.3714 \\
\hline House $(512 \times 512)$ & 0.8949 & 48.6130 & 0.8156 & 49.0160 \\
\hline
\end{tabular}


It is known that PSNR values indicate an objective quality measure for an image processing system performance. They do not indicate any subjective measure. Subjective evaluation of the reconstructed images needs some visual inspection of these images to investigate artifacts and to measure their perceptual quality.

Scores of similarity are obtained for the three test images by calculating the average mean opinion score $(M O S)$ [19] of 20 observers. It is known that $4<M O S \leq 5$ indicates no distortion (Excellent), $3<M O S \leq 4$ represents little distortions which can be ignored (Good), $2<M O S \leq 3$ shows clear distortions which can be seen but can be accepted (Fair), $\quad 1<M O S \leq 2$ shows a lot of distortion which can't be accepted (Bad) and finally $\quad 0<M O S \leq 1$ shows too much distortion, therefore cannot be tolerated (Very Bad). Table 4 shows that excellent MOS values are achieved for the three reconstructed images of Fig. 9 while applying the proposed bio 9/7 wavelet filters rather than the original ones.

Table 4 MOS values for the test images using original and proposed bio 9/7 wavelet filters.

\begin{tabular}{|c|c|c|}
\hline Image & $\begin{array}{c}\text { MOS values using original } \\
\text { bio } 9 / 7 \text { wavelet filters }\end{array}$ & $\begin{array}{c}\text { MOS values using the proposed } \\
\text { bio } 9 / 7 \text { wavelet filters }\end{array}$ \\
\hline Lena $(512 \times 512)$ & 4.6 & 4.7 \\
\hline Peppers $(512 \times 512)$ & 4.7 & 4.8 \\
\hline House $(512 \times 512)$ & 4.4 & 4.6 \\
\hline
\end{tabular}

\section{Conclusions}

A simple and efficient design for the filter bank of the symmetric bio 9/7 DWT has been introduced with less computations. A modification in the standard design constraints of the lifting scheme has led to sets of simple and reduced-number filter coefficients. Some interesting characteristics regarding those coefficients have been investigated to ease the overall filter bank realization. Those coefficients have been realized in a SOPOT multiplier-less manner. The comparison of the resulting multiplier-free structure with some of other recent multiplier-free structures has proven its superiority in utilizing a minimum number of different basic building blocks of filters. The realized symmetric bio 9/7 DWT has been tested through analyzing and synthesizing some standard images.

While testing the designed structure, excellent objective and subjective quality measures have been achieved compared to the original bio 9/7 DWT filter bank. Such excellent quality measures highlight the suitability of the proposed structure for image processing. In addition, since the proposed design has applied the standard design constraints of the lifting scheme, it is believed that the resulting structure will also be very efficient in many other applications such as image/speech compression and denoising.

The proposed design can be extended further via McClellan transformation to some efficient special types of 2-D wavelet filter banks, such as; circular-symmetric, ellipticalsupport, and fan filters. The later can also be utilized in designing efficient contourlet transforms. 


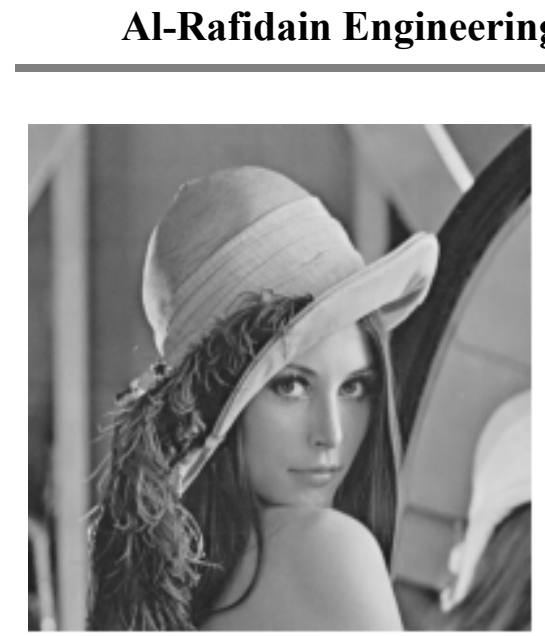

(a)

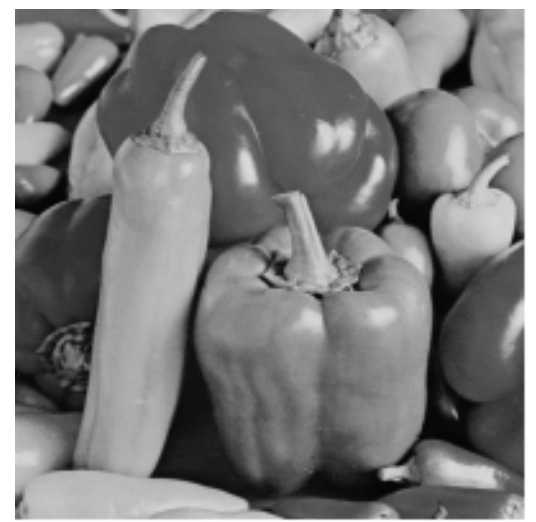

(d)

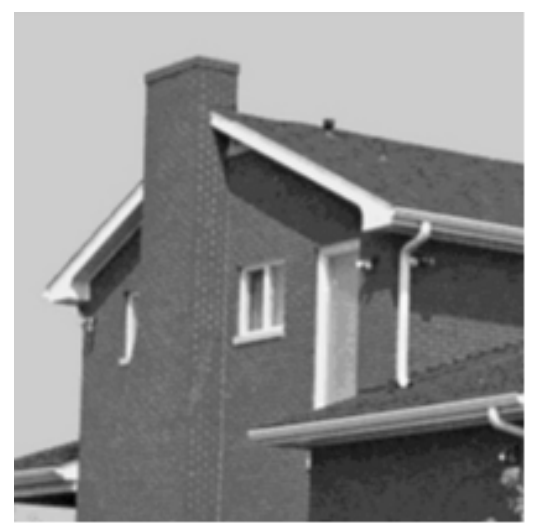

(g)

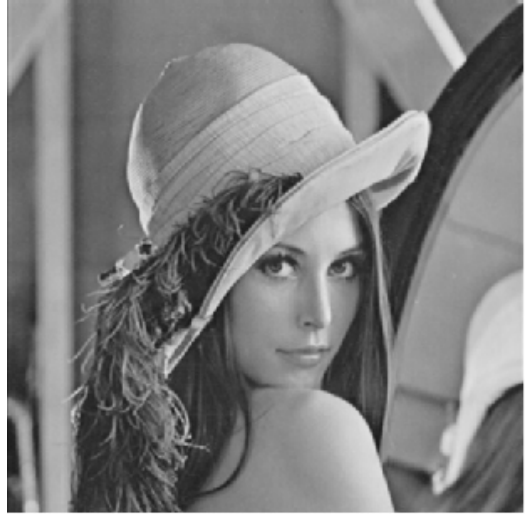

(b), $M O S=4.6$

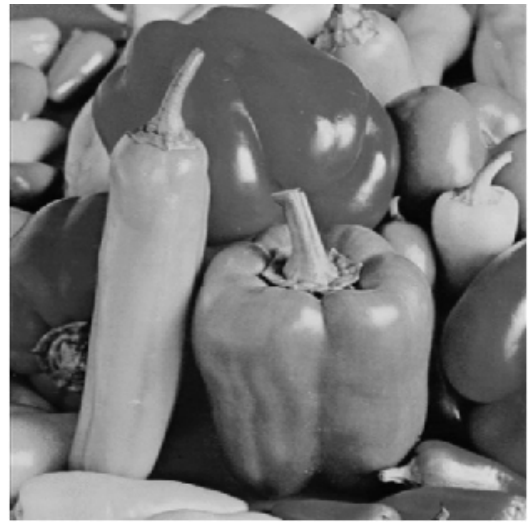

(e), MOS = 4.7

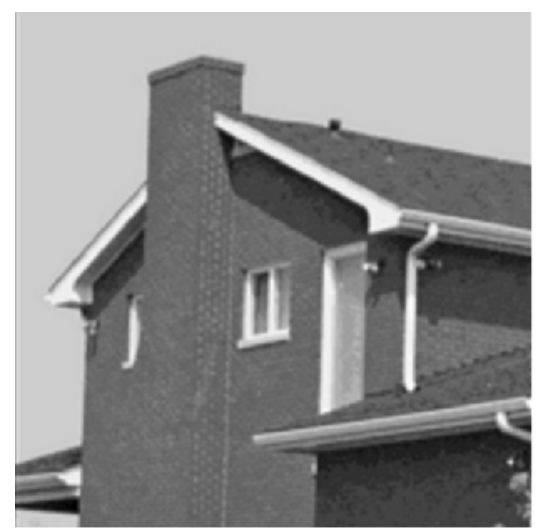

(h), $M O S=4.4$
No. 3

June 2015

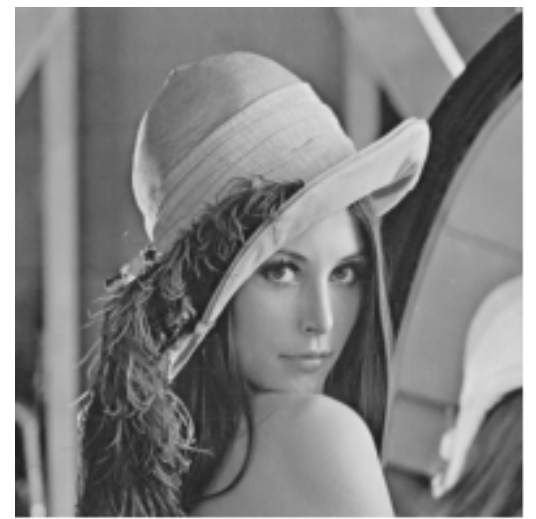

(c), $M O S=4.7$

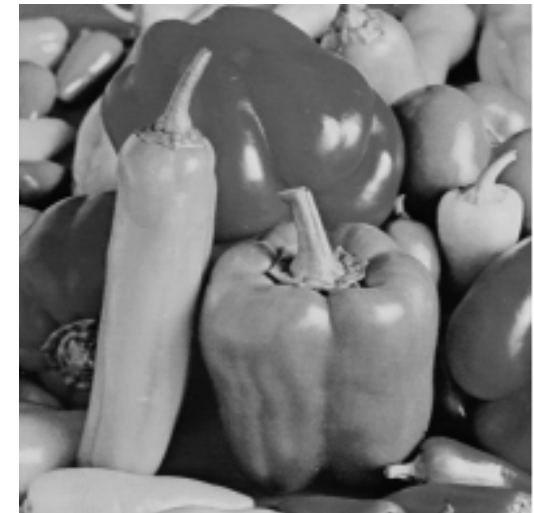

(f), $M O S=4.8$

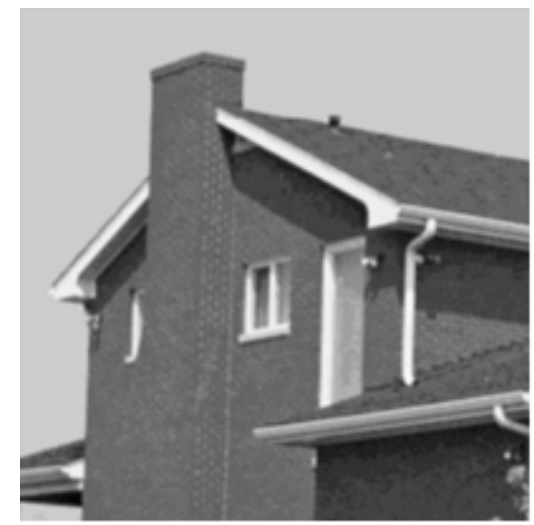

(i), $M O S=4.6$

Fig. 9 The three test images and their reconstructions using original and proposed bio 9/7 filter banks with the corresponding MOS values, (a) Original Lena image, (b) and (c) Reconstructed Lena images using original and proposed bio 9/7 filter bank, respectively, (d) Original Peppers image, (e) and (f) Reconstructed Peppers images using original and proposed bio 9/7 filter bank, respectively, (g) Original House image, (h) and (i) Reconstructed House images using original and proposed bio 9/7 filter bank, respectively. 


\section{References}

[1] C. Liu, Y. Liu, Z. Qiu and X. Du, "On The Initialization of The Discrete Wavelet Transform Algorithm", IEEE International Conference on Intelligent Processing Systems, Beijing, China, 28 - 31 Oct 1997, Vol. 2, pp. 1220 - 1222.

[2] J. M. Abdul-Jabbar, Z. R. Al-Omari, R. W. Hmad and N. M. Al-Lila, " A Novel Design and FPGA Implementation of The Biorthogonal 5/3 Filter Bank", Al-Rafidain Engineering Journal, College of Engineering, University of Mosul, Vol. 21, No. 4, Aug. 2013, pp. 87-95.

[3] Chapter 4, Implementation of DWT Filter Banks, Fayetteville State University, North Carolina ، ETD 11242003:

http://www.coursehero.com/file/1837263/11dschapter4/

[4] J. M. Abdul-Jabbar, and Z. T. Abed Al-Mokhtar, "Design and FPGA Implementation of Two-Dimensional Discrete Wavelet Transform Architectures Using Raster-Scan Method", Al-Rafidain Engineering Journal, College of Engineering, University of Mosul, Vol. 22, No. 2, March 2014, pp. 69-72.

[5] M. Martina, and G. Masera, , "Low-Complexity, Efficient 9/7 Wavelet Filters VLSI implementation", IEEE Transactions on Circuits and Systems II: Express Briefs, Vol. 53 , No. 11, Nov. 2006, pp. 1289 - 1293.

[6] M. Beladgham, A. Bessaid, A. M. Lakhdar, and A. Taleb-Ahmed, "Improving Quality of Medical Image Compression Using Biorthogonal CDF Wavelet Based on Lifting Scheme and SPIHT Coding, Serbian Journal Of Electricatl Engineering Vol. 8, No. 2, May 2011, pp. 163-179.

[7] S. Zhang, G. Yang, Z. Cheng, and H. van de Wetering, "A Novel 9/7 Wavelet Filter banks For Texture Image Coding", International Journal of Advanced Research in Artificial Intelligence, Vol. 1, No. 6, 2012, pp. 7-14.

[8] K. A. Kotteri, S. Barua, A. E. Bell, and J. E. Carletta, "A Comparison of Hardware Implementations of the Biorthogonal 9/7 DWT: Convolution Versus Lifting", IEEE Trans. Circuits Systems II, express briefs, Vol. 52, No. 5, May 2005, pp. $256-260$.

[9] L. Cheng, D. L. Liang, and Z. H. Zhang, "Popular Biorthogonal Wavelet Filters Via A Lifting Scheme and Its Application in Image Compression," IEE Proceedings Vision, Image and Signal Processing, Vol.150, 2003, pp. 227-232.

[10] A. Z. Averbuch and V. A. Zheludev, "A New Family of Spline-Based Biorthogonal Wavelet Transforms and Their Application to Image Compression, IEEE Transactions on Image Processing, Vol. 13, No. 7, July 2004, pp. 993- 1007.

[11] Do Quan, and Yo-Sung Ho, "Efficient Wavelet Lifting Scheme Based on Filter Optimization and Median Operator," IEEE-RIVF International Conference on Computing and Communication Technologies, Da Nang Univ Technol, Da Nang, Vietnam, 2009, pp. 284-289.

[12] T. Strutz, "Wavelet Filter Design based on the Lifting Scheme and its Application in Lossless Image Compression”, WSEAS Transactions on Signal Processing, Vol. 5, No. 2, Feb. 2009, pp. 53- 62.

[13] G. Tewari, S. Sardar, and K. A. Babu, "High-Speed \& Memory Efficient 2-D DWT on Xilinx Spartan3A DSP using Scalable Polyphase Structure with DA for JPEG2000 Standard", 3rd International Conference on Electronics Computer Technology (ICECT), Kanyakumari, Vol. 1, 8-10 April 2011, pp. 138 - 142. 
[14] A. P. Gupta, S. Singh, and N. Meena, "Low Power and Area Efficient 2-D DWT Using 9/7 Filter based on NEDA Technique, International Journal of Computer Applications, Vol.80, No. 15, Oct. 2013, pp. 18-21.

[15] K. A. Kotteri, "Optimal, Multiplierless Implementations of the Discrete Wavelet Transform for Image Compression Applications", M. Sc. Thesis submitted to the Faculty of the Virginia Polytechnic Institute and State University, 2004.

[16] K. Andra, C. Chakrabarti and T. Acharya, "A VLSI architecture for lifting based forward and inverse wavelet transform" IEEE Transaction on Signal Processing, Vol. 50, No. 4, April 2002, pp. $966-977$.

[17] B. M. Lutovac and M. D. Lutovac, "Design and VHDL Description of Multiplierless Half-Band IIR Filter", International Journal of Electronics and Communications (AEU), Vol. 56, No. 5, October 2002, pp. 348 - 350.

[18] R. Guy, "Unsolved Problems in Number Theory, 3rd ed., New York: SpringerVerlag, 2010.

[19] A. Saffor, A. bin Ramli, K. Ng and D. Dowsett, "Objective and Subjective Evaluation of Compressed Computed Tomography (CT) Images", The Internet Journal of Medical Simulation, Vol. 1, No. 12001. 